Alitéia Santiago Dilélio'

Elaine Tomasi"

Elaine Thumé

Denise Silva da Silveira ${ }^{\text {Iv }}$

Fernando Carlos Vinholes Siqueira $^{\mathrm{V}}$

Roberto Xavier Piccinilv

Suele Manjourany Silva"

Bruno Pereira Nunes"

Luiz Augusto Facchini"

Departamento de Ciências da Saúde. Universidade Federal de Santa Maria. Palmeira das Missões, RS, Brasil

" Programa de Pós-Graduação em Epidemiologia. Universidade Federal de Pelotas. Pelotas, RS, Brasil

III Programa de Pós-Graduação em Enfermagem. Universidade Federal de Pelotas. Pelotas, RS, Brasil

Iv Faculdade de Medicina. Universidade Federal de Pelotas. Pelotas, RS, Brasil

Programa de Pós-Graduação em Educação Física. Universidade Federal de Pelotas. Pelotas, RS, Brasil

Correspondence:

Alitéia Santiago Dilélio

Departamento de Medicina Social - UFPEL Av. Duque de Caxias, $2503^{\circ}$ andar Fragata 96030-001 Pelotas, RS, Brasil

E-mail: aliteia@gmail.com

Received: 4/6/2014

Approved: 9/23/2014

Article available from: www.scielo.br/rsp

\section{Lack of access and continuity of adult health care: a national population-based survey}

\author{
Falta de acesso e de continuidade \\ da atenção à saúde em adultos: \\ inquérito nacional
}

\begin{abstract}
OBJECTIVE: To describe the lack of access and continuity of health care in adults.

METHODS: A cross-sectional population-based study was performed on a sample of 12,402 adults aged 20 to 59 years in urban areas of 100 municipalities of 23 states in the five Brazilian geopolitical regions. Barriers to the access and continuity of health care and were investigated based on receiving, needing and seeking health care (hospitalization and accident/emergency care in the last 12 months; care provided by a doctor, by other health professional or home care in the last three months). Based on the results obtained by the description of the sample, a projection is provided for adults living in Brazilian urban areas.
\end{abstract}

RESULTS: The highest prevalence of lack of access to health services and to provision of care by health professionals was for hospitalization (3.0\%), whilst the lowest prevalence was for care provided by a doctor $(1.1 \%)$. The lack of access to care provided by other health professionals was $2.0 \%$; to accident and emergency services, $2.1 \%$; and to home care, $2.9 \%$. As for prevalences, the greatest absolute lack of access occurred in emergency care (more than 360,000 adults). The main reasons were structural and organizational problems, such as unavailability of hospital beds, of health professionals, of appointments for the type of care needed and charges made for care.

CONCLUSIONS: The universal right to health care in Brazil has not yet been achieved. These projections can help health care management in scaling the efforts needed to overcome this problem, such as expanding the infrastructure of health services and the workforce.

DESCRIPTORS: Adult. Health Services Accessibility. Continuity of Patient Care. Health Inequalities. Equity in Health. Health Surveys. 


\section{RESUMO}

OBJETIVO: Descrever a falta de acesso e de continuidade da atenção à saúde de adultos.

MÉTODOS: Estudo transversal de base populacional com 12.402 adultos entre 20 e 59 anos, residentes em áreas urbanas de 100 municípios de 23 estados brasileiros, nas cinco regiões geopolíticas. Investigaram-se as barreiras no acesso e na continuidade da atenção a partir do recebimento, necessidade e busca de algum atendimento de saúde (internação hospitalar e pronto-socorro nos 12 meses prévios ao estudo; atendimento médico, de outro profissional de saúde e domiciliar nos três meses prévios). A partir dos resultados obtidos na descrição da amostra, apresenta-se uma projeção para os adultos residentes em áreas urbanas no território nacional.

RESULTADOS: A prevalência de falta de acesso aos serviços e aos atendimentos com profissionais de saúde mais expressiva foi de $3,0 \%$, para internação hospitalar, enquanto a menor prevalência foi para atendimento médico $(1,1 \%)$. A falta de acesso para o atendimento com outro profissional de saúde foi de 2,0\%; em pronto-socorro, 2,1\%; e domiciliar, 2,9\%. Quanto às prevalências, o maior número absoluto de falta de acesso foi para atendimentos de urgência (mais de 360.000 adultos). Os principais motivos foram problemas estruturais e organizacionais, como falta de leito, de profissionais, de ficha/vaga do tipo de atendimento necessário e cobrança pelo atendimento.

CONCLUSÕES: O direito universal à saúde no Brasil ainda não foi alcançado. As projeções podem apoiar a gestão no dimensionamento de esforços dirigidos ao seu enfrentamento, como a ampliação da estrutura física dos serviços e da força de trabalho.

DESCRITORES: Adulto. Acesso aos Serviços de Saúde. Continuidade da Assistência ao Paciente. Desigualdades em Saúde. Equidade em Saúde. Inquéritos Epidemiológicos.

\section{INTRODUCTION}

The Brazilian Unified Health System (SUS) is guided by the principles of universal and equal access and aims to provide services based on health needs. ${ }^{20,21}$

The concept of "access" is complex and multidimensional, and may focus on people's characteristics, service supply or the relationship between people and services. ${ }^{19}$ In this study, the concept is a synonym of accessibility, referring to the possibility of using health services when necessary ${ }^{18}$ Lack of access is the impossibility of using the service regardless of need, expressing lack of capacity to respond or difficulty in ensuring health care. ${ }^{22}$

The behavioural model proposed by Andersen and Newman identifies access as one of the mediators of health service use, and indicates four dimensions of access: potential access, referring to contextual factors; realized access, relating to service use; effective access, referring to the care process; and efficient access, focusing on changes in health conditions and satisfaction as a result of the received care. ${ }^{1,18}$

The mechanisms that regulate the seeking and use of health services need to be understood, such as health needs which do or do not lead to services being sought; when seeking care, demand is generated and this is mediated by the existence or inexistence of the service, the main barrier to access. ${ }^{18}$ Other barriers are geographic distribution, the availability and quality of human and technical resources and the health care model. ${ }^{20}$

According to Pereira, ${ }^{16}$ when health services exist, needs may transform into demand for services and their use. A health need is "any disturbance to health or well-being, from both the patient's and the health professional's point of view, capable of resulting in a demand on the health system". Needs can also be perceived (when they are identified by the individual), or unperceived (when they are only identified by a health professional). ${ }^{16}$ 
Even when the need is felt, an individual has "freedom of use" based on the degree of information about choices or, in other words, an individual has a health need but does not seek care owing to personal decisions. ${ }^{18}$

Continuity of care also deserves attention, and its quality must be evaluated. Continuity is related to the experiences and relationship of a patient with a clinician, as well as coordinated clinical care as the patient moves between different parts of the health service. ${ }^{11}$ Adequate diagnosis and treatment procedures culminating in user satisfaction would be the desired course for all health care. ${ }^{7,14}$

The individual's lack of access to first contact or the lack of continuity of care can be considered indicators of the quality of health services by identifying inadequacies as: shortage of professionals, setting and fixed days for performing programmatic actions shifts.

Despite the importance of barriers to access to health services and to continuity of care regarding public health and impact on health indicators, studies in this area are incipient. This study aimed to describe lack of access and lack of health care continuity for adults.

\section{METHODS}

This study was part of a survey that investigated the access and quality of health services in Brazil. This was a cross-sectional population-based study performed on a sample of 12,402 adults with ages from 20 to 59 years, resident in urban areas of 100 cities in 23 states in the five Brazilian geopolitical regions. ${ }^{9}$

The sample size was estimated a posteriori based on prevalence rates. The largest sample required had 9,151 subjects, assuming: $0.7 \%$ prevalence of lack of access, accuracy of $0.2 \mathrm{pp}$, and a design effect of 1.2 , including $15.0 \%$ for losses and refusals. It described the characteristics of continuous care.

The sample representing the adult urban population was located by a multiple level sampling process ${ }^{9,17}$ that considered population size, census tracts and households. Municipalities were aggregated according to population size and selected systematically. Census tracts were allocated randomly according to the proportion of valid sectors and population size, using the official grid of the 2000 Population Census. ${ }^{a}$ Ten households were visited in each of the 638 sectors selected, "jumping" systematically 30 households from one residence to the next with the aim of finding 17 adults per sector.
All adults resident in the households were eligible to be interviewed. The 55 selected and trained interviewers collected the data using a hand-held device (PDA - personal digital assistant) in 2009. The data was stored on portable computers and transferred to the study headquarters via Internet.

The questionnaire was standardized and pre-tested. It contained socioeconomic and demographic variables as well as variables regarding both public and private health service use.

The respondents were asked if they had received some form of health care recently (hospital admission and accident/emergency care in the last 12 months; care provided by a doctor, other health professional or domiciliary care in the last three months) in order to investigate lack of access. If no, they were asked whether they had needed it, despite not having had care (yes/no). The sequence of questions asked about care provided by a doctor can be exemplified as follows: "In the <last three months $>$, have you been seen by a doctor?" If no: "Despite not having been seen by a doctor, did you need to be seen by one?" If needed: "Have you sought the care of a doctor in the <last three months $>$ ?" If the care of a doctor was sought: "Why were you not seen by a doctor?". The questions for investigating the other forms of care provision followed the same pattern. Care provided by other health professional referred to top-level professionals, including dentists, psychologists, physiotherapists, dieticians, physical education teachers, speech therapists, social workers and nurses.

The reason for not receiving care and the outcome of the problem were investigated to describe barriers to access. With regard to care provided by a doctor, another health professional or domiciliary care, the respondents were asked whether they had sought care and the reason why they had not sought care. The reason why care was needed was also considered when it was provided by a doctor or domiciliary care.

In order to characterize barriers to care continuity, the respondents were asked about: all forms of care provided, the outcome of the problem after receiving care, whether they had received any explanation about the reason for care being provided, whether they were referred for a follow-up appointment, whether the follow-up appointment took place and the reason why they did not have their follow-up appointment. They were also asked whether they were referred to another service, whether any of these referrals did not take place and the reason why they did not have the care to which they had been referred regarding care provided by a doctor, another health professional or domiciliary care. Exclusively in relation to care provided by a

\footnotetext{
a Instituto Brasileiro de Geografia e Estatística. Censo brasileiro de 2000. Rio de Janeiro; 2000 [cited 2010 mar 10]. Available from:
} http://www.ibge.gov.br/home/estatistica/populacao/censo2000/default_prim_resultados.shtm 
doctor, respondents were asked whether any tests had been requested and the reason for not having them. The term "continuity of care" was used instead of "management continuity", since part of the visits may refer to a continuum of care throughout life, and part may be related to specific problems. ${ }^{10,11}$

The descriptive variables were gender (male/female), self-reported skin color (white; black; other - mixed, Asian and indigenous categories), age in years (20 to 29; 30 to $39 ; 40$ to $49 ; 50$ to 59 ), education in years (none; one to four; five to eight; nine to $11 ; 12$ or more), economic classification as per Brazilian Association of Research Companies $(\mathrm{ABEP})^{\mathrm{b}}$ (A and $\mathrm{B} ; \mathrm{C}$; D and $\left.\mathrm{E}\right)$, per capita income in minimum wages $(\leq 0.3 ; 0.4$ to $<1 ; 1.0$ and more), morbidity diagnosed medically (hypertension; diabetes mellitus; nerve problems - yes/no), geopolitical region (MW/N/NE/SE/S) and municipality population size $(\leq 30,000 ; 30,001$ to 100,$000 ; 100,001$ to $1,000,000$; $>1,000,000$ inhabitants).

For the purposes of quality control, $5.0 \%$ of the interviews were selected randomly to be repeated within three days at most, following the first interview. Hospitalization in the last year and a medical reference hypertension resulted kappa index of 0.77 and 0.73 , respectively.

Based on the results obtained by the sample, a projection was provided for the reference population, i.e. adults in urban areas throughout Brazil, based on a total of $92,168,985$ inhabitants. $^{c}$

Analysis was performed using the Stata 12.1 statistics package.

This study was approved by the Research Ethics Committee of the Faculdade de Medicina of Universidade Federal de Pelotas (Process 152/07). All respondents signed an informed consent form.

\section{RESULTS}

A total of 13,756 adults were identified. Losses and refusals accounted for $9.8 \%$, resulting in 12,402 individuals included in the study; $55.1 \%$ were women and $39.7 \%$ self-reported white skin colour. Average age was 37.4 years $(\mathrm{SD}=11.5$ years $)$ and $31.8 \%$ were aged 20 to 29 years.

Approximately $1 / 3$ of the individuals had between nine and 11 years of schooling. Most individuals fell into economic classification C (51.5\%) and $42.0 \%$ had per capita income $>1$ minimum wage. A quarter of the respondents reported medical diagnosis of at least one chronic disease. The highest number of respondents lived in the Southeast (35.4\%), in cities with between 100,001 and 1,000,000 inhabitants (39.9\%).

About 3.0\% were not admitted and did not even have access to hospitalization (223,324 adult Brazilians), among those who self-reported need to be admitted to a hospital. The majority needed to be admitted to a hospital for an emergency surgery $(30.0 \%)$ or to undergo examinations (20.0\%); 40.0\% did not admit themselves to a hospital because they thought they did not need to; and $20.0 \%$ reported that the outcome of their health problem was worsened (Table 1).

Of those adults who were admitted to a hospital, $42.9 \%$ were discharged without being referred for a follow-up appointment and $20.6 \%$ of those who were referred did not actually have the appointment (4.7\% for difficulties within the health service: $1.9 \%$ - no appointments available; $0.9 \%$ - no doctor available onsite; $1.9 \%$ - lack of SUS doctors) (Table 1).

Of individuals seeking accident/emergency care, $2.1 \%$ (360,186 people) did not receive it. Almost half (49.0\%) gave up waiting to be seen since it took too long and the problem causing them to seek care had got worse in $16.3 \%$ of cases (Table 2 ).

The majority $(85.1 \%)$ provided with accident/emergency care were discharged without being referred for a follow-up appointment and $28.4 \%$ of those who were referred did not attend it. Lack of access to follow-up appointments owing to difficulties within the health service (no appointments available (1.1\%); no doctor available onsite (5.5\%); lack of SUS doctor (2.2\%)) accounted for $8.8 \%$ (Table 2).

Out of all respondents who sought care provided by a doctor, $1.1 \%$ did not receive care $(n=49): 353,867$ Brazilian adults were estimated not to have had access. The main reason for needing to be seen by a doctor, even though they were not seen, was "because I thought I needed to, I was feeling ill" (80.8\%). Reasons relating to health promotion and prevention were reported by $53.9 \%$ of the sample: health problem follow-up/monitoring $(29.9 \%)$, check-up $(17.4 \%)$, pre-cancerous conditions $(4.1 \%)$, prostate examination $(1.5 \%)$ and antenatal appointment (1.0\%). Around $70.7 \%$ of individuals who reported needing care actually sought it. The main reasons for this were the absence of a doctor (42.6\%) and unavailability of appointments (40.4\%). When asked why they had not sought care provided by a doctor, $53.4 \%$ stated difficulty in getting an appointment on SUS and $9.3 \%$ the lack of a doctor. The majority $(50.3 \%)$ considered that their health problem

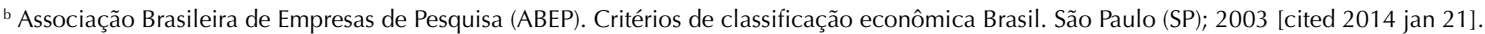
Available from: http://www.abep.org

c Instituto Brasileiro de Geografia e Estatística. Censo brasileiro de 2010. Rio de Janeiro (RJ); 2010 [cited 2014 jan 21] Available from: http://censo2010.ibge.gov.br/
} 
Table 1. Description of barriers to access and continued care based on the indicated need for hospital admission among adults. Brazil, 2009.

\begin{tabular}{|c|c|c|c|c|c|}
\hline \multirow[t]{2}{*}{ Variable (n) } & \multicolumn{3}{|c|}{ Sample } & \multicolumn{2}{|c|}{$\begin{array}{l}\text { Projection for the urban population } \\
(20 \text { to } 59 \text { years })^{\text {b }} 92,168,985\end{array}$} \\
\hline & $\mathrm{n}^{\mathrm{a}}$ & $\%$ & $95 \% \mathrm{Cl}$ & $\mathrm{n}$ & $95 \% \mathrm{Cl}$ \\
\hline Hospitalized $(\mathrm{n}=12,365)$ & 961 & 7.8 & $7.3 ; 8.3$ & $7,189,181$ & $6,728,335 ; 7,757,856$ \\
\hline $\begin{array}{l}\text { Despite not being hospitalized, a doctor had indicated } \\
\text { hospitalization }(n=11,404)\end{array}$ & 30 & 0.3 & & & \\
\hline \multicolumn{6}{|c|}{ Barriers to access } \\
\hline Lack of access to hospitalization $(\mathrm{n}=991)^{\mathrm{c}}$ & 30 & 3.0 & $2.0 ; 4.3$ & 223,324 & 148,$882 ; 320,097$ \\
\hline \multicolumn{6}{|l|}{ Reason for needing to be hospitalized $(n=30)$} \\
\hline Emergency surgery/Operation & 9 & 300 & $14.7 ; 49.4$ & 76,482 & 37,$476 ; 125,940$ \\
\hline Non-emergency surgery/Operation & 2 & 6.7 & $8.2 ; 22.1$ & 17,081 & 20,$905 ; 56,342$ \\
\hline Clinical treatment & 7 & 23.3 & $9.9 ; 42.3$ & 59,401 & 25,$239 ; 107,839$ \\
\hline To have tests/Examinations & 6 & 20.0 & $7.7 ; 38.6$ & 50,988 & 19,$630 ; 98,407$ \\
\hline Other & 6 & 20.0 & $7.7 ; 38.6$ & 50,988 & 19,$630 ; 98,407$ \\
\hline \multicolumn{6}{|l|}{ Reason for not being hospitalized $(n=30)$} \\
\hline Thought they did not need it & 12 & 40.0 & $22.7 ; 59.4$ & 101,976 & 57,$871 ; 151,434$ \\
\hline No hospital bed available & 6 & 20.0 & $7.7 ; 38.6$ & 50,988 & 19,$630 ; 98,407$ \\
\hline Family or work commitments & 5 & 16.7 & $5.6 ; 34.7$ & 42,575 & 14,$277 ; 88,464$ \\
\hline Afraid & 4 & 13.3 & $3.7 ; 30.7$ & 33,907 & 9,$433 ; 78,266$ \\
\hline Unable to pay & 3 & 10.0 & $2.1 ; 26.5$ & 25,494 & 5,$354 ; 67,559$ \\
\hline No transport available & 1 & 3.3 & $0.08 ; 17.2$ & 8,413 & $204 ; 43,850$ \\
\hline Other & 6 & 20.0 & $7.7 ; 38.6$ & 50,988 & 9,$630 ; 98,407$ \\
\hline \multicolumn{6}{|l|}{ Health problem outcome after not being hospitalized $(\mathrm{n}=30)$} \\
\hline Got worse & 6 & 20.0 & $7.7 ; 38.6$ & 50,988 & 19,$630 ; 98,407$ \\
\hline Just the same as before & 12 & 40.0 & $22.7 ; 59.4$ & 101,976 & 57,$871 ; 151,434$ \\
\hline Improved a little & - & - & - & & \\
\hline Improved considerably & 4 & 13.3 & $3.7 ; 30.7$ & 33,907 & 9,$433 ; 78,266$ \\
\hline Cured/Problem solved & 8 & 26.7 & $12.3 ; 45.9$ & 68,069 & 31,$358 ; 117,017$ \\
\hline \multicolumn{6}{|c|}{ Barriers to continuity of care } \\
\hline $\begin{array}{l}\text { Did not receive explanation as to the reason for being } \\
\text { hospitalized }(n=915)\end{array}$ & 799 & 87.3 & $85.0 ; 89.4$ & $6,276,155$ & $6,110,804 ; 6,427,128$ \\
\hline $\begin{array}{l}\text { Discharged from hospital without being referred to a } \\
\text { follow-up appointment }(\mathrm{n}=935)\end{array}$ & 401 & 42.9 & $39.7 ; 46.1$ & $3,084,159$ & $2,854,105 ; 3,314,212$ \\
\hline Did not have the follow-up appointment $(n=534)$ & 110 & 20.6 & $17.2 ; 24.3$ & 845,635 & 706,$064 ; 997,520$ \\
\hline \multicolumn{6}{|l|}{ Reason why did not have follow-up appointment $(n=108)$} \\
\hline Date of the appointment not reached yet & 59 & 54.6 & $44.7 ; 64.2$ & 461,716 & 377,$999 ; 542,897$ \\
\hline Did not attempt to make an appointment & 33 & 30.6 & $22.1 ; 40.2$ & 258,764 & 186,$885 ; 339,945$ \\
\hline Unable to go and make the appointment & 3 & 2.8 & $0.6 ; 7.9$ & 23,678 & 5,$074 ; 66,805$ \\
\hline Attempted to make an appointment. but none available & 2 & 1.9 & $0.2 ; 6.5$ & 16,067 & 1,$691 ; 54,966$ \\
\hline $\begin{array}{l}\text { No doctor available on SUS and unable to pay for a } \\
\text { private doctor }\end{array}$ & 2 & 1.9 & $0.2 ; 6.5$ & 16,067 & 1,$691 ; 54,966$ \\
\hline $\begin{array}{l}\text { Attempted to make an appointment, but no doctor } \\
\text { available at that service }\end{array}$ & 1 & 0.9 & $0.02 ; 5.0$ & 7,611 & $169 ; 42,282$ \\
\hline Other & 8 & 7.4 & $3.3 ; 14.1$ & 62,577 & 27,$906 ; 119,234$ \\
\hline \multicolumn{6}{|l|}{ Opinion about care received $(n=923)$} \\
\hline Very bad & 43 & 4.7 & $3.4 ; 6.2$ & 337,891 & 244,$432 ; 445,729$ \\
\hline Poor & 12 & 1.3 & $0.7 ; 2.3$ & 93,459 & 5,$032 ; 165,351$ \\
\hline Regular & 79 & 8.6 & $6.8 ; 10.6$ & 618,270 & 488,$864 ; 762,053$ \\
\hline
\end{tabular}


Continuation

\begin{tabular}{lrrrrc}
\hline Good & 448 & 48.5 & $45.3 ; 51.8$ & $3,486,753$ & $3,256,699 ; 3,723,996$ \\
Very good & 341 & 36.9 & $33.8 ; 40.2$ & $2,652,808$ & $2,429,943 ; 2,890,051$ \\
What happened to the problem after being hospitalized $(\mathrm{n}=885)$ & & & & \\
Got worse & 18 & 2.0 & $1.2 ; 3.2$ & 143,784 & 86,$270 ; 230,054$ \\
Just the same as before & 59 & 6.7 & $5.1 ; 8.5$ & 481,675 & 366,$648 ; 611,080$ \\
Improved a little & 140 & 15.8 & $13.5 ; 18.4$ & $1,135,891$ & 970,$539 ; 1,322,809$ \\
Improved considerably & 258 & 29.2 & $26.2 ; 32.3$ & $2,099,241$ & $1,883,565 ; 2,322,105$ \\
Cured/Problem solved & 410 & 46.3 & $43.0 ; 49.7$ & $3,328,591$ & $3,091,348 ; 3,573,0.23$ \\
\hline
\end{tabular}

SUS: Brazilian Unified Health System

${ }^{a}$ Information is partially unknown for some variables. Differing values may therefore appear.

${ }^{\mathrm{b}}$ Instituto Brasileiro de Geografia e Estatística, Censo Demográfico, 2010.

${ }^{\mathrm{c}}$ The denominator refers to the total of individuals who were hospitalized plus individuals who reported having medical indication for hospitalization but did not have access to it.

Table 2. Description of barriers to access and continued care based on adults' self-reported need for accident and emergency care. Brazil, 2009.

\begin{tabular}{|c|c|c|c|c|c|}
\hline \multirow[t]{2}{*}{ Variable $(n)$} & \multicolumn{3}{|c|}{ Sample } & \multicolumn{2}{|c|}{$\begin{array}{c}\text { Projection for the urban population } \\
(20 \text { to } 59 \text { years })^{b} 92,168,985\end{array}$} \\
\hline & $\mathrm{n}^{\mathrm{a}}$ & $\%$ & $95 \% \mathrm{Cl}$ & $\mathrm{n}$ & $95 \% \mathrm{Cl}$ \\
\hline Accident and Emergency Care $(\mathrm{n}=12,302)$ & 2,243 & 18.2 & $17.6 ; 18.9$ & $16,774,755$ & $16,221,741 ; 17,419,938$ \\
\hline Needed care, despite not receiving it $(n=10,059)$ & 49 & 0.5 & & & \\
\hline \multicolumn{6}{|c|}{ Barriers to access } \\
\hline Lack of access to accident and emergency care $(n=2,292)^{c}$ & 49 & 2.1 & $1.6 ; 2.8$ & 360,186 & 274,$428 ; 480,248$ \\
\hline \multicolumn{6}{|l|}{ Reason why care was not provided $(n=49)$} \\
\hline Waiting time too long, gave up & 24 & 49.0 & $34.4 ; 63.7$ & 184716 & 129,$678 ; 240,131$ \\
\hline Too many people waiting & 18 & 36.7 & $23.4 ; 51.7$ & 138,348 & 88,$211 ; 194,894$ \\
\hline Specialist not available & 12 & 24.5 & $13.3 ; 38.9$ & 92,358 & 50,$137 ; 146,642$ \\
\hline Service refused to provide care & 8 & 16.3 & $7.3 ; 29.7$ & 61,446 & 27,$519 ; 111,960$ \\
\hline No transport available & 2 & 4.1 & $0.5 ; 14.0$ & 15,456 & $188 ; 52,776$ \\
\hline Other & 10 & 20.0 & $10.2 ; 34.3$ & 75,394 & 38,$451 ; 129,301$ \\
\hline \multicolumn{6}{|l|}{ Problem outcome after not receiving care $(n=49)$} \\
\hline Got worse & 8 & 16.3 & $7.3 ; 29.7$ & 61,446 & 27,$519 ; 111,960$ \\
\hline Just the same as before & 15 & 30.6 & $18.3 ; 45.4$ & 115,353 & 68,$986 ; 171,145$ \\
\hline Improved a little & 14 & 28.6 & $16.6 ; 43.3$ & 107,814 & 62,$577 ; 163,229$ \\
\hline Improved considerably & 7 & 14.3 & $5.9 ; 27.2$ & 53,907 & 22,$241 ; 102,536$ \\
\hline Cured/Problem solved & 5 & 10.2 & $3.4 ; 22.2$ & 38,451 & 12,$817 ; 83,688$ \\
\hline \multicolumn{6}{|c|}{ Barriers to continuity of care } \\
\hline $\begin{array}{l}\text { Did not receive an explanation as to the reason for seeking } \\
\text { care }(n=2,126)\end{array}$ & 1.415 & 66.6 & $64.5 ; 68.6$ & $11,171,987$ & $10,819,717 ; 11,507,482$ \\
\hline $\begin{array}{l}\text { Discharged from the accident and emergency unit without } \\
\text { being referred to a follow-up appointment }(n=2,178)\end{array}$ & 1.854 & 85.1 & $83.6 ; 86.6$ & $14,275,317$ & $14,023,695 ; 14,526,938$ \\
\hline Did not have the follow-up appointment $(\mathrm{n}=324)$ & 92 & 28.4 & $23.6 ; 33.6$ & 709,841 & 589,$867 ; 839,811$ \\
\hline \multicolumn{6}{|l|}{ Reason why did not have the follow-up appointment $(n=91)$} \\
\hline Did not attempt to make an appointment & 33 & 36.3 & $26.4 ; 47.0$ & 257,672 & 187,$398 ; 333,625$ \\
\hline Date of the appointment not reached yet & 29 & 31.9 & $22.5 ; 42.5$ & 226,439 & 159,$714 ; 301,682$ \\
\hline $\begin{array}{l}\text { Attempted to make an appointment, but no doctor } \\
\text { available at that service }\end{array}$ & 5 & 5.5 & $1.8 ; 12.4$ & 39,041 & 12,$777 ; 88,020$ \\
\hline Unable to go and make the appointment & 3 & 3.3 & $0.7 ; 9.3$ & 23,425 & 4,$969 ; 66,015$ \\
\hline
\end{tabular}

Continue 


\begin{tabular}{lccccc} 
Continuation & & & \\
\hline $\begin{array}{l}\text { No doctor available on SUS and unable to pay for a private } \\
\text { doctor }\end{array}$ & 2 & 2.2 & $0.3 ; 7.7$ & 15,616 & 2,$130 ; 54,658$ \\
Attempted to make an appointment, but none available & 1 & 1.1 & $0.03 ; 6.0$ & 7,808 & $213 ; 42,590$ \\
Other & 18 & 19.8 & $12.2 ; 29.5$ & 140,548 & 86,$601 ; 209,403$ \\
Opinion about care received $(\mathrm{n}=2,083)$ & & & & & \\
Very bad & 183 & 8.8 & $7.6 ; 10.1$ & $1,476,178$ & $1,274,881 ; 1,694,250$ \\
Poor & 99 & 4.8 & $3.9 ; 5.8$ & 805,188 & 654,$215 ; 972,936$ \\
Regular & 352 & 16.9 & $15.3 ; 18.6$ & $2,834,934$ & $2,566,538 ; 3,120,104$ \\
Good & 1.105 & 53.0 & $50.9 ; 55.2$ & $8,890,620$ & $8,538,350 ; 9,259,665$ \\
Very good & 344 & 16.5 & $14.9 ; 18.2$ & $2,767,835$ & $2,415,656 ; 3,053,005$ \\
Problem outcome after care was provided $(\mathrm{n}=2,158)$ & & & & & \\
Got worse & 48 & 2.2 & $1.6 ; 2.9$ & 369,045 & 268,$396 ; 486,468$ \\
Just the same as before & 341 & 15.8 & $14.3 ; 17.4$ & $2,650,411$ & $2,398,790 ; 2,918,807$ \\
Improved a little & 584 & 27.1 & $25.2 ; 29.0$ & $4,545,959$ & $4,227,238 ; 4,864,679$ \\
Improved considerably & 669 & 31.0 & $29.1 ; 33.0$ & $5,200,174$ & $4,881,454 ; 5,535,669$ \\
Cured/Problem solved & 516 & 23.9 & $22.1 ; 25.8$ & $4,009,167$ & $3,707,221 ; 4,327,887$ \\
\hline
\end{tabular}

SUS: Brazilian Unified Health System

a Information is partially unknown for some variables. Differing values may therefore appear.

b Instituto Brasileiro de Geografia e Estatística, Censo Demográfico, 2010.

${ }^{c}$ The denominator refers to the total of individuals who received care in an accident and emergency unit plus individuals who reported needing this type of care but did not have access to it.

continued just the same after having been seen by the doctor (Table 3).

More than half the adults (57.1\%) left the appointment without being referred for tests after being seen by a doctor, $16.4 \%$ did not have any tests performed because they were not able to on the SUS or because they were not available in their city (4.4\%). Many (92.9\%) left the appointment without being referred to care at another service (Table 3 ).

Of the respondents, $2.0 \%$ stated that although they had not received care provided by a health professional other than a doctor, they needed it (representing 246,417 adults). More than half (56.0\%) reported not having sought care, especially for the difficulty in getting an appointment on SUS (57.1\%). The main reasons why adults did not receive care were the lack of appointments $(27.3 \%)$ and users being unable to pay for services $(24.2 \%)$. The majority $(61.3 \%)$ stated that their health problem remained unaltered (Table 4).

Most individuals (95.9\%) who received care were not referred to other services. Around $41.0 \%$ of those who were referred did not receive the care to which they had been referred either because they were not able to get this care on SUS $(20.0 \%)$ or because it was not available in their city $(4.0 \%)$ (Table 4$)$.

Lack of access to domiciliary care was reported by $2.9 \%$ of the adults (representing 126,447 individuals). Transport difficulties $(35.5 \%)$, being confined to bed $(29.0 \%)$, blood pressure problems $(16.1 \%)$ and backache $(12.9 \%)$ were the most reported reasons for needing to receive domiciliary care. Approximately $3 / 4$ reported not having requested domiciliary care. The majority $(47.1 \%)$ did not receive care because, even though they sought it, no response was provided by the health service, the health service had no professional available to provide the care $(47.1 \%)$, or the health service did not provide this kind of care $(41.2 \%)$. The most mentioned reasons for not seeking domiciliary care were because the health service did not provide this kind of service $(68.9 \%)$ and there was no professional available to provide it $(5.6 \%)$. The majority $(45.2 \%)$ stated that their condition remained the same as before seeking care (Table 5).

Around $93.6 \%$ of those who did receive domiciliary health care were not referred to other services, and $11.4 \%$ of those who were referred did not reach these other services because they were unable to schedule an appointment (25.0\%) (Table 5).

\section{DISCUSSION}

The prevalence of lack of access to services and provision of care by health professionals was relatively low and showed little variation. Lack of access was more prevalent $(3.0 \%)$ for individuals who reported needing hospitalization, whilst individuals who reported needing provision of care by a doctor presented the lowest prevalence $(1.1 \%)$. The prevalence of lack of 
Table 3. Description of barriers to access and continued care based on adults' self-reported need to be seen by a doctor. Brazil, 2009.

\begin{tabular}{|c|c|c|c|c|c|}
\hline \multirow[t]{2}{*}{ Variable (n) } & \multicolumn{3}{|c|}{ Sample } & \multicolumn{2}{|c|}{$\begin{array}{l}\text { Projection for the urban population } \\
\text { (20 to } 59 \text { years })^{b} 92,168,985\end{array}$} \\
\hline & $\mathrm{n}^{\mathrm{a}}$ & $\%$ & $95 \% \mathrm{Cl}$ & $\mathrm{n}$ & $95 \% \mathrm{Cl}$ \\
\hline Care provided by a doctor $(\mathrm{n}=12,300)$ & 4,241 & 34.5 & $33.6 ; 35.3$ & $31,798,300$ & $30,968,779 ; 32,535,652$ \\
\hline Needed care, despite not receiving it $(n=8,059)$ & 167 & 2.1 & & & \\
\hline \multicolumn{6}{|c|}{ Barriers to access } \\
\hline \multicolumn{6}{|l|}{ Reason why needed care $(n=167)$} \\
\hline Thought they needed it because felt unwell & 135 & 80.8 & $74.0 ; 86.5$ & $1,024,370$ & 938,$160 ; 1,096,633$ \\
\hline Follow-up on health problem & 50 & 29.9 & $23.1 ; 37.5$ & 379,068 & 292,$858 ; 475,419$ \\
\hline To have a check-up & 29 & 17.4 & $12.0 ; 24.0$ & 220,594 & 152,$134 ; 304,268$ \\
\hline To request tests/Examinations & 19 & 11.4 & $7.0 ; 17.2$ & 144,527 & 88,$745 ; 218,059$ \\
\hline To request a prescription & 8 & 4.8 & $2.1 ; 9.2$ & 60,854 & 26,$623 ; 116,636$ \\
\hline Cancer screening $(\mathrm{n}=98)$ & 4 & 4.1 & $1.1 ; 10.1$ & 51,979 & 13,$946 ; 128,046$ \\
\hline To request a medical certificate & 3 & 1.8 & $0.4 ; 5.2$ & 22820 & 5,$071 ; 65,925$ \\
\hline To receive the results of tests & 3 & 1.8 & $0.4 ; 5.2$ & 22,820 & 5,$071 ; 65,925$ \\
\hline Prostate examination $(n=69)$ & 1 & 1.5 & $0.03 ; 7.8$ & 19,017 & $380 ; 98,887$ \\
\hline To have an antenatal appointment $(\mathrm{n}=98)$ & 1 & 1.0 & $0.03 ; 5.6$ & 12,678 & $380 ; 70,996$ \\
\hline Other & 12 & 7.2 & $3.8 ; 12.2$ & 91,280 & 48,$176 ; 154,670$ \\
\hline Lack of access to care provided by a doctor $(n=4,290)^{c}$ & 49 & 1.1 & $0.8 ; 1.5$ & 353,867 & 257,$358 ; 482,546$ \\
\hline Did not attempt to make an appointment $(n=167)$ & 118 & 70.7 & $63.1 ; 77.4$ & 896,324 & 799,$972 ; 981,265$ \\
\hline \multicolumn{6}{|l|}{$\begin{array}{l}\text { Reason why did not attempt to make an appointment } \\
(\mathrm{n}=118)\end{array}$} \\
\hline Difficulty in getting an appointment on the SUS & 63 & 53.4 & $44.0 ; 62.6$ & 478,637 & 394,$382 ; 561,099$ \\
\hline Afraid/Did not want to & 34 & 28.8 & $20.9 ; 37.9$ & 258,141 & 187,$332 ; 339,707$ \\
\hline Family or work commitments & 25 & 21.2 & $14.2 ; 29.7$ & 190,021 & 127,$278 ; 266,208$ \\
\hline Unable to pay & 24 & 20.4 & $13.5 ; 28.7$ & 182,850 & 121,$004 ; 257,245$ \\
\hline Unable to go and make appointment & 18 & 15.3 & $9.3 ; 23.0$ & 137,138 & 83,$358 ; 206,154$ \\
\hline No doctor available at their usual health service & 11 & 9.3 & $4.7 ; 16.1$ & 83,358 & 42,$127 ; 144,308$ \\
\hline Health complaint got better & 5 & 4.2 & $1.4 ; 9.6$ & 37,646 & 12,$549 ; 86,047$ \\
\hline Other & 17 & 14.5 & $8.6 ; 22.1$ & 129,967 & 77,$084 ; 198,088$ \\
\hline \multicolumn{6}{|l|}{ Reason why care was not provided $(n=47)$} \\
\hline No doctor available & 20 & 42.6 & $28.3 ; 57.8$ & 158,242 & 105,$123 ; 214,704$ \\
\hline No appointment available & 19 & 40.4 & $26.4 ; 55.7$ & 150,070 & 98,$066 ; 206,904$ \\
\hline Unable to pay & 7 & 14.9 & $6.2 ; 28.3$ & 55,348 & 23,$031 ; 105,123$ \\
\hline Service closed when care was sought & 1 & 2.1 & $0.05 ; 11.3$ & 7,801 & $186 ; 41,975$ \\
\hline \multicolumn{6}{|l|}{ Problem outcome after not receiving care $(n=167)$} \\
\hline Got worse & 17 & 10.2 & $6.0 ; 15.8$ & 129,314 & 76,$067 ; 200,310$ \\
\hline Just the same as before & 84 & 50.3 & $42.5 ; 58.1$ & 637,696 & 538,$808 ; 736,583$ \\
\hline Improved a little & 31 & 18.5 & $13.0 ; 25.3$ & 234,540 & 164,$812 ; 320,749$ \\
\hline Improved considerably & 21 & 12.6 & $8.0 ; 18.6$ & 159,741 & 101,$423 ; 235,808$ \\
\hline Cured/Problem solved & 14 & 8.4 & $4.7 ; 13.7$ & 106,494 & 59,$586 ; 173,686$ \\
\hline
\end{tabular}

Barriers to continuity of care

\begin{tabular}{lllll}
\hline Did not receive an explanation as to the reason for $\quad 3.035$ & 74.0 & $72.7 ; 75.4$ & $23,530,742$ & $23,117,364 ; 23,975,918$
\end{tabular} seeking care $(n=4,100)$

Appointment ended without tests having been requested $(n=4,159)$

$2.376 \quad 57.1 \quad 55.6 ; 58.6 \quad 18,156,829 \quad 17,679,855 ; 18,633,804$

Reason(s) why did not have some of the test(s) requested $(n=526)$

Appointment made, but has not happened yet

$\begin{array}{lllll}279 & 53.0 & 48.7 ; 57.4 & 7,229,979 & 6,643,396 ; 7,830,204\end{array}$

Continue 


\begin{tabular}{|c|c|c|c|c|c|}
\hline \multicolumn{6}{|c|}{ Continuation } \\
\hline Unable to get tests on the SUS and unable to pay & 86 & 16.4 & $13.4 ; 19.8$ & $2,237,201$ & $1,827,957 ; 2,701,011$ \\
\hline Unable to go and make appointment/Have tests & 53 & 10.1 & $7.6 ; 13.0$ & $1,377,789$ & $1,036,752 ; 1,773,391$ \\
\hline Did not want to & 41 & 7.8 & $5.7 ; 10.4$ & $1,064,035$ & 777,$564 ; 1,418,713$ \\
\hline Not available in their city & 23 & 4.4 & $2.8 ; 6.5$ & 600,225 & 381,$961 ; 886,696$ \\
\hline Thought they did not need them & 22 & 4.2 & $2.6 ; 6.3$ & 572,942 & 354,$678 ; 859,413$ \\
\hline Other & 82 & 15.6 & $12.6 ; 19.0$ & $2,128,069$ & $1,718,825 ; 2,591,879$ \\
\hline $\begin{array}{l}\text { Appointment ended without being referred to another } \\
\text { service }(n=4,178)\end{array}$ & 3.881 & 92.9 & $92.1 ; 93.7$ & $29,540,621$ & $29,286,234 ; 29,795,007$ \\
\hline Did not go to some of the referrals $(n=294)$ & 100 & 34.0 & $28.6 ; 39.7$ & 767,611 & $645,696,276 ; 896,298,677$ \\
\hline \multicolumn{6}{|l|}{ Reason why did not go to some of the referrals $(n=100)$} \\
\hline Appointment made, but has not happened yet & 45 & 45.0 & $35.0 ; 55.3$ & 345,425 & 268,$664 ; 24,489$ \\
\hline Unable to go and make appointment/Have them & 12 & 12.0 & $6.4 ; 20.0$ & 92,113 & 49,$127 ; 153,522$ \\
\hline Unable to get tests on the SUS and unable to pay & 11 & 11.0 & $5.6 ; 18.8$ & 84,437 & 42,$986 ; 144,311$ \\
\hline Did not want to & 9 & 9.0 & $4.2 ; 16.4$ & 69,085 & 32,$240 ; 125,888$ \\
\hline Thought they did not need them & 4 & 4.0 & $1.1 ; 9.9$ & 30,704 & 8,$444 ; 75,993$ \\
\hline Not available in their city & 3 & 3.0 & $0.6 ; 8.5$ & 23,028 & 4,$606 ; 65,247$ \\
\hline Other & 22 & 22.0 & $14.3 ; 31.4$ & 168,874 & 109,$768 ; 241,030$ \\
\hline \multicolumn{6}{|l|}{ Opinion about care received $(n=4,027)$} \\
\hline Very bad & 107 & 2.7 & $2.2 ; 3.2$ & 858,554 & 699,$563 ; 1,017,546$ \\
\hline Poor & 61 & 1.5 & $1.2 ; 1.9$ & 476,974 & 381,$580 ; 604,168$ \\
\hline Regular & 313 & 7.8 & $7.0 ; 8.6$ & $2,480,267$ & $2,225,881 ; 2,734,654$ \\
\hline Good & 2.169 & 53.8 & $52.3 ; 55.4$ & $17,107,485$ & $16,630,511 ; 17,616,258$ \\
\hline Very good & 1.377 & 34.2 & $32.7 ; 35.7$ & $10,875,019$ & $10,398,044 ; 11,351,993$ \\
\hline \multicolumn{6}{|l|}{ Problem outcome after care was provided $(n=4,000)$} \\
\hline Got worse & 41 & 1.0 & $0.7 ; 1.4$ & 317,983 & 222,$588 ; 445,176$ \\
\hline Just the same as before & 1.293 & 32.3 & $30.9 ; 33.8$ & $10,270,851$ & $9,825,675 ; 10,747,825$ \\
\hline Improved a little & 848 & 21.2 & $19.9 ; 22.5$ & $6,741,240$ & $6,327,862 ; 7,154,617$ \\
\hline Improved considerably & 1.046 & 26.2 & $24.8 ; 27.5$ & $8,331,155$ & $7,885,978 ; 8,744,532$ \\
\hline Cured/Problem solved & 772 & 19.3 & $18.1 ; 20.6$ & $6,137,072$ & $5,755,492 ; 6,550,450$ \\
\hline
\end{tabular}

SUS: Brazilian Unified Health System

${ }^{a}$ Information is partially unknown for some variables. Differing values may therefore appear.

b Instituto Brasileiro de Geografia e Estatística, Censo Demográfico, 2010.

'The denominator refers to the total of individuals who had care provided by a doctor plus individuals who sought this type of care but did not have access to it.

access to care provided by a health professional other than a doctor, lack of access to accident/emergency care and lack of access to domiciliary care was $2.0 \%, 2.1 \%$ and $2.9 \%$, respectively.

Data from the 2003 National Household Sample Survey (PNAD) shows that the prevalence of lack of access among adults aged 20 to 64 years who sought health services in the last two weeks was of $2.2 \%$. The 2008
PNAD found $2.8 \%$ for this same item. ${ }^{\mathrm{d}, \mathrm{e}}$ The prevalence rates of this study are similar to those found in PNAD, even though the PNAD respondents' recollection period was shorter, their age range was greater and more subjects were interviewed than in this study.

A study conducted in the city of Pelotas, Southern Brazil, found a $6.5 \%$ prevalence rate of lack of access to a health service in the last month in a sample of adults

d Instituto Brasileiro de Geografia e Estatística. Pesquisa Nacional por Amostra de Domicílios - PNAD: acesso e utilização dos serviços de saúde 2003. Rio de Janeiro (RJ); 2005 [cited 2014 Dec 8]. Available from: http://www.spm.gov.br/arquivos-diversos/.arquivos/integra_saude_ibge_2003 e Instituto Brasileiro de Geografia e Estatística. Pesquisa Nacional por Amostra de Domicílios - PNAD: um panorama da saúde no Brasil: acesso e utilização dos serviços, condições de saúde e fatores de risco e proteção à saúde 2008. Rio de Janeiro (RJ); 2008 [cited 2014 Dec 8]. Suplemento. Available from: http://www.ibge.gov.br/home/estatistica/populacao/panorama_saude_brasil_2003_2008/ 
Table 4. Description of barriers to access and continued care based on adults' self-reported need to have care provided by a health professional other than a doctor. Brazil, 2009.

\begin{tabular}{|c|c|c|c|c|c|}
\hline \multirow[t]{2}{*}{ Variable (n) } & \multicolumn{3}{|c|}{ Sample } & \multicolumn{2}{|c|}{$\begin{array}{l}\text { Projection for the urban population } \\
(20 \text { to } 59 \text { years })^{b} 92,168,985\end{array}$} \\
\hline & $\mathrm{n}^{\mathrm{a}}$ & $\%$ & IC95\% & $\mathrm{n}$ & IC95\% \\
\hline $\begin{array}{l}\text { Care provided by professional other than a doctor } \\
(\mathrm{n}=12,279)\end{array}$ & 1,61 & 13.1 & $12.5 ; 13.8$ & $12,074,137$ & $11,521,123 ; 12,719,320$ \\
\hline Needed care, despite not receiving it $(n=10,666)$ & 75 & 0.7 & & & \\
\hline \multicolumn{6}{|c|}{ Barriers to access } \\
\hline $\begin{array}{l}\text { Lack of access to health professional other than a doctor } \\
(\mathrm{n}=1,646)^{\mathrm{c}}\end{array}$ & 33 & 2.0 & $1.4 ; 2.8$ & 246,417 & 178,$070 ; 356,141$ \\
\hline Did not make an appointment $(n=75)$ & 42 & 56.0 & $44.1 ; 67.5$ & 313,972 & 247,$253 ; 378,448$ \\
\hline \multicolumn{6}{|l|}{ Reason why did not make appointment $(n=42)$} \\
\hline Difficulty in getting an appointment on the SUS & 24 & 57.1 & $41.0 ; 72.3$ & 179,278 & 128,$728 ; 227,002$ \\
\hline Unable to pay & 12 & 28.6 & $15.7 ; 44.6$ & 89,796 & 49,$294 ; 140,031$ \\
\hline Family or work commitments & 12 & 28.6 & $15.7 ; 44.6$ & 89,796 & 49,$294 ; 140,031$ \\
\hline Unable to go and make appointment & 9 & 21.4 & $10.3 ; 36.8$ & 67,190 & 32,$339 ; 115,542$ \\
\hline $\begin{array}{l}\text { This type of professional not available at their usual } \\
\text { health service }\end{array}$ & 8 & 19.0 & $8.6 ; 34.1$ & 59,655 & 27,$002 ; 107,064$ \\
\hline Afraid/Did not want to & 6 & 14.3 & $5.4 ; 28.5$ & 44,898 & 16,$954 ; 89,482$ \\
\hline Health complaint got better & 1 & 2.4 & $0.06 ; 12.6$ & 7,535 & $188 ; 39,560$ \\
\hline Other & 2 & 4.8 & $0.6 ; 16.2$ & 15,071 & 1,$884 ; 50,863$ \\
\hline \multicolumn{6}{|l|}{ Reason why care was not provided $(\mathrm{n}=33)$} \\
\hline No appointment available & 9 & 27.3 & $13.3 ; 45.5$ & 67,347 & 32,$810 ; 112,245$ \\
\hline Unable to pay & 8 & 24.2 & $11.1 ; 42.3$ & 59,699 & 27,$383 ; 104,351$ \\
\hline This type of professional not available at the service & 5 & 15.4 & $5.1 ; 31.9$ & 37,991 & 12,$581 ; 78,695$ \\
\hline Service closed when care was sought & 1 & 3.0 & $0.08 ; 15.8$ & 7,401 & $197 ; 38,977$ \\
\hline Other & 11 & 33.3 & $18.0 ; 51.8$ & 82,148 & 44,$405 ; 127,787$ \\
\hline \multicolumn{6}{|l|}{ Problem outcome after not receiving care $(\mathrm{n}=75)$} \\
\hline Got worse & 16 & 21.3 & $12.7 ; 32.3$ & 119,421 & 71,$204 ; 181,094$ \\
\hline Just the same as before & 46 & 61.3 & $49.4 ; 72.4$ & 343,687 & 276,$968 ; 405,921$ \\
\hline Improved a little & 10 & 13.3 & $6.6 ; 23.2$ & 74,568 & 34,$004 ; 130,074$ \\
\hline Improved considerably & 1 & 1.3 & $0.03 ; 7.2$ & 7,289 & $168 ; 40,368$ \\
\hline Cured/Problem solved & 2 & 2.7 & $0.3 ; 9.3$ & 15,138 & 1,$682 ; 52,142$ \\
\hline
\end{tabular}

Barriers to continuity of care

Appointment ended without being referred to another service $(n=1,537)$

Did not go to some of the referrals $(n=61)$

$\begin{array}{lllll}1.474 & 95.9 & 94.8 ; 96.8 & 11,579,097 & 11,446,282 ; 11,687,765\end{array}$

Reason why did not go to some of the referrals $(n=25)$

Appointment made, but has not happened yet

Unable to get tests on the SUS and unable to pay

Did not want to

25

$25 \quad 41.0 \quad 28.6 ; 54.3 \quad 202,966 \quad 141,581 ; 268,807$

Thought they did not need them

Unable to go and make appointment/Have them

Not available in their city

Other

3

(1)

Opinion about care received $(n=1,492)$

Very bad

Continue 


\begin{tabular}{lccccc} 
Continuation & & & & & \\
\hline Poor & 14 & 1.0 & $0.5 ; 1.6$ & 120,741 & 60,$371 ; 193,186$ \\
Regular & 65 & 4.3 & $3.4 ; 5.5$ & 519,188 & 410,$521 ; 664,078$ \\
$\quad$ Good & 758 & 50.8 & $48.2 ; 53.4$ & $6,133,662$ & $5,819,734 ; 6,447,589$ \\
$\quad$ Very good & 639 & 42.8 & $40.3 ; 45.4$ & $5,167,731$ & $4,865,877 ; 5,481,658$ \\
Problem outcome after care was provided ( $\mathrm{n}=1,466)$ & & & & & \\
$\quad$ Got worse & 13 & 0.9 & $0.5 ; 1.5$ & 108,667 & 60,$371 ; 181.112$ \\
Just the same as before & 271 & 18.5 & $16.5 ; 28.6$ & $2,233,715$ & $1,992,233 ; 3,453,203$ \\
Improved a little & 234 & 16.0 & $14.1 ; 17.9$ & $1,931,862$ & $1,702,453 ; 2,161,271$ \\
Improved considerably & 500 & 34.1 & $31.7 ; 36.6$ & $4,117,281$ & $3,827,501 ; 4,419,134$ \\
Cured/Problem solved & 448 & 30.5 & $28.2 ; 33.0$ & $3,682,612$ & $3,404,907 ; 3,984,465$ \\
\hline
\end{tabular}

SUS: Brazilian Unified Health System

a Information is partially unknown for some variables. Differing values may therefore appear.

${ }^{\mathrm{b}}$ Instituto Brasileiro de Geografia e Estatística. Censo Demográfico, 2010.

' The denominator refers to the total of individuals who received care from a health professional other than a doctor plus individuals who sought this type of care but did not have access to it.

aged 20 or more. The inclusion of older adults may have led to the higher percentage found by that study. ${ }^{\mathrm{f}}$

Despite being low, the prevalence rates found in this study reach another dimension when they are extrapolated to include all adults living in urban areas in Brazil. Access to a doctor, for example, is estimated to be lacking for more than 350,000 public and private health service users and 360,000 adults are estimated to be without access to emergency care. This is worsened by the service being mainly public. These projections are expected to be useful in supporting health management to calculate the efforts needed to address it, such as scaling up the physical structure of the services and the workforce.

Similarity was found among the reasons for seeking care by both those who did and those who did not manage to access services, with regard to hospitalization, care provided by a doctor and domiciliary care (data not presented). Different from the 2003 PNAD, which found preventive actions to be the main reason for health care provision, our study identified surgical operations and illnesses in general as being the main reasons for needing to be admitted to a hospital or needing to receive health care, like the 2008 PNAD. ${ }^{e}$

The main reason for lack of access by those seeking health care was structural and organizational problems: lack of hospital beds, charges for services, having to wait a long time to be seen, lack of health professionals, lack of appointments availability and lack of the kind of care needed. Similar aspects were found by the PNAD and by the study conducted in Pelotas, RS, in particular charges for services, unavailability of appointments and lack of doctors. ${ }^{\mathrm{f}}$
Difficulty in getting an appointment on SUS, financial and personal problems and unavailability of services were the reasons for not seeking domiciliary care $(72.6 \%)$, care provided by a doctor $(70.7 \%)$ and care provided by other health professionals $(56.0 \%)$. Lack of access can precede health care seeking, whereby service users identify barriers beforehand. A similar characteristic can be seen in barriers for having tests done.

The 2003 PNAD found that the main barriers to access were financial (23.8\%) and long waiting times (18.1\%).g Financial and structural problems continue to be significant obstacles to accessing health services. Service users can think it is so unlikely for them to get an appointment at their health centre within a short space of time that the majority do not even try to get one. ${ }^{5}$

Geographical, financial, organizational, information and cultural barriers to access are an expression of the characteristics of supply that can facilitate or impede people's ability to use services. Travassos \& Castro ${ }^{20}$ highlight the most important barrier as being the unavailability or physical absence of services and human resources. However, information about such availability, the illness in question and treatment options are also important in facilitating the use of health services, as these factors have an impact on people's perception of health. Individual perception can also affect use of services, as both the quest for and use of them can be triggered by perceived need. ${ }^{18}$

Despite the important progress made by SUS, inequalities in universal access still exist. Problems relating to equity and comprehensiveness persist in that structural aspects reported as being the main barriers to accessing health services and ensuring continuity of care. ${ }^{15,23}$

\footnotetext{
${ }^{\dagger}$ Nunes BP. Acesso aos serviços de saúde em adolescentes e adultos na cidade de Pelotas - RS [dissertation]. Pelotas (RS): Universidade Federal de Pelotas, Departamento de Medicina Social; 2012.

$\mathrm{g}$ Instituto Brasileiro de Geografia e Estatística. Pesquisa Nacional por Amostra de Domicílios - PNAD: acesso e utilização dos serviços de saúde 2003. Rio de Janeiro (RJ); 2005 [cited 2014 Dec 8]. Available from: http://www.spm.gov.br/arquivos-diversos/.arquivos/integra_saude_ibge_2003
} 
Table 5. Description of barriers to access and continued care based on adults' self-reported need for domiciliary care. Brazil, 2009.

\begin{tabular}{lccccc}
\hline \multirow{2}{*}{ Variable $(\mathrm{n})$} & \multicolumn{3}{c}{\begin{tabular}{c} 
Sample \\
\cline { 2 - 6 }
\end{tabular}} & & \multicolumn{2}{c}{$\begin{array}{c}\text { Projection for the urban population } \\
(20 \text { to } 59 \text { years) }\end{array}$} \\
\cline { 2 - 6 } & $\mathrm{n}^{\mathrm{a}}$ & $\%$ & $95 \% \mathrm{Cl}, 168,985$ & $\mathrm{n}$ & $95 \% \mathrm{Cl}$ \\
\hline Domiciliary care $(\mathrm{n}=12,281)$ & 560 & 4.6 & $4.2 ; 4.9$ & $4,239,773$ & $3,871,097 ; 4,516,280$ \\
Needed care at home, despite not receiving it $(\mathrm{n}=11,721)$ & 62 & 0.5 & & & \\
\hline
\end{tabular}

Barriers to access

Reason why needed care $(\mathrm{n}=62)$

Transport difficulties

Confined to bed

Blood pressure problem

Had backache

Had leakage/Stroke/Ischaemia

Had a mental problem

Had rheumatism/Joint problems

Had heart problems

Had a neurological problem

Needed to get vaccinated

Had diabetes

Had been hospitalized in the last three months

Had had surgery recently

Had cancer

Had injured limbs or back

Lack of access to domiciliary care $(\mathrm{n}=577)^{c}$

Did not request domiciliary care $(n=62)$

Reason why did not request domiciliary care $(n=45)$

Service does not provide domiciliary care

No professional available to provide domiciliary care

Health complaint got better

Unable to go and make an appointment or request

domiciliary care

Afraid/Did not want to

Service telephone always engaged or not working

Service does not have telephone

Other

Reason why domiciliary care was not provided $(n=17)$

Sought care but health service did not reply

Sought care but health service did not have a professional available

Sought care but health service does not provide this type of care

Sought care but no appointment available

No SUS professional available and unable to pay privately

Sought care but the service was closed

Telephone always engaged $(n=17)$

Other $(\mathrm{n}=17)$

Problem outcome after not receiving care $(n=62)$

Got worse
Just the same as before

$\begin{array}{lll}10 & 16.1 & 8.0 ; 27.7\end{array}$

70,783

$28 \quad 45.2 \quad 32.5 ; 58.3 \quad 198,720$
35,$172 ; 121,782$

04,$196 ; 214,108$

80,$016 ; 184,651$

35,$172 ; 121,782$

25,060;105,075

11,$870 ; 78,257$

11,$870 ; 78,257$

11,$870 ; 78,257$

7,$914 ; 69,024$

440;59,352

440;59,352

1,$759 ; 49,240$

1,$759 ; 49,240$

1,$759 ; 49,240$

$176 ; 38,249$

74,$124 ; 204,931$

262,$908 ; 365,786$

170,444;261,092

127,$673 ; 224,705$

20,747;94,159

16,$278 ; 85,541$

11,$810 ; 76,923$

1,$596 ; 48,516$

$192 ; 37,664$

16,$278 ; 85,541$

27,$706 ; 8,717,527$

27,$706 ; 8,717,527$

22,$165 ; 8,101,746$

17,$106 ; 7,449,743$

8,191;6,037,069

$120 ; 3,465,277$

142,$885 ; 256,314$

Continue 


\begin{tabular}{|c|c|c|c|c|c|}
\hline \multicolumn{6}{|l|}{ Continuation } \\
\hline Improved a little & 11 & 17.7 & $9.2 ; 29.5$ & 77,817 & 40,$447 ; 129,696$ \\
\hline Improved considerably & 10 & 16.1 & $8.0 ; 27.7$ & 70,783 & 35,$172 ; 121,782$ \\
\hline Cured/Problem solved & 3 & 4.8 & $1.0 ; 13.5$ & 21,103 & 4,$396 ; 59,352$ \\
\hline \multicolumn{6}{|c|}{ Barriers to continuity of care } \\
\hline $\begin{array}{l}\text { Did not receive an explanation as to the reason for seeking } \\
\text { care }(n=537)\end{array}$ & 444 & 82.7 & $79.2 ; 85.8$ & $3,506,293$ & $3,357,900 ; 3,637,725$ \\
\hline Received care but not referred to any other service $(n=550)$ & 515 & 93.6 & $91.3 ; 95.5$ & $3,968,428$ & $3,870,913 ; 4,048,984$ \\
\hline Did not go to some of the referrals $(n=35)$ & 4 & 11.4 & $3.2 ; 26.7$ & 30,933 & 8,$683 ; 72,449$ \\
\hline \multicolumn{6}{|l|}{ Reason why did not go to some of the referrals $(n=4)$} \\
\hline Appointment made. but has not happened yet & 1 & 25.0 & $0.6 ; 80.6$ & 7,733 & $186 ; 24,932$ \\
\hline Unable to get tests on SUS and unable to pay & - & - & - & - & - \\
\hline Thought they did not need them & 1 & 25.0 & $0.6 ; 80.6$ & 7,733 & $186 ; 24,932$ \\
\hline Did not want to & 1 & 25.0 & $0.6 ; 80.6$ & 7,733 & $186 ; 24,932$ \\
\hline Unable to go and make appointment/Have them & 1 & 25.0 & $0.6 ; 80.6$ & 7,733 & $186 ; 24,932$ \\
\hline Not available in their city & - & - & - & - & - \\
\hline Other & 1 & 25.0 & $0.6 ; 80.6$ & 7,733 & $186 ; 24,932$ \\
\hline \multicolumn{6}{|l|}{ Opinion about care received $(n=548)$} \\
\hline Very bad & 3 & 0.6 & $0.1 ; 1.6$ & 25,439 & 4,$240 ; 67,836$ \\
\hline Poor & 5 & 0.9 & $0.3 ; 2.1$ & 38,158 & 12,$719 ; 89,035$ \\
\hline Regular & 46 & 8.4 & $6.2 ; 11.0$ & 356,141 & 262,$866 ; 466,375$ \\
\hline Good & 350 & 63.8 & $59.7 ; 67.9$ & $2,704,975$ & $2,531,145 ; 2,878,806$ \\
\hline Very good & 144 & 26.3 & $22.6 ; 30.2$ & $1,115,060$ & 958,$189 ; 1,280,412$ \\
\hline \multicolumn{6}{|l|}{ Problem outcome after care was provided $(n=427)$} \\
\hline Got worse & 1 & 0.2 & $0.006 ; 1.3$ & 8,480 & $254 ; 55,117$ \\
\hline Just the same as before & 222 & 52.0 & $47.1 ; 56.8$ & $2,204,682$ & $1,996,933 ; 2.408,191$ \\
\hline Improved a little & 52 & 12.2 & $9.2 ; 15.7$ & 517,252 & 390,$059 ; 665,644$ \\
\hline Improved considerably & 99 & 23.2 & $19.3 ; 27.5$ & 983,627 & 818,$276 ; 1,165,938$ \\
\hline Cured/Problem solved & 53 & 12.4 & $9.4 ; 15.9$ & 525,732 & 398,$539 ; 674,124$ \\
\hline
\end{tabular}

SUS: Brazilian Unified Health System

${ }^{a}$ Information is partially unknown for some variables. Differing values may therefore appear.

${ }^{\mathrm{b}}$ Instituto Brasileiro de Geografia e Estatística. Censo Demográfico, 2010.

${ }^{c}$ The denominator refers to the total of individuals who received domiciliary care plus individuals who sought this type of care but did not have access to it.

Longitudinality, i.e. service users being accompanied over time by health professionals, is considered to be a key characteristic of Primary Health Care. However, this concept can also be applied to other levels of care, given that it relates to positive health care results and can be used to assess its quality. ${ }^{7,10}$

There is a lack of information about the need for or indication by health professionals to have follow-up after care, about the need to have tests and the need to be referred to another health professional. Despite that, continuity of care and assessment of the service user's condition can result in more accurate diagnosis and more efficacious treatment, in addition to optimizing referrals to specialists and the performance of more complex procedures. The same can be observed regarding the prescription of tests and medication, especially about the lack of access to some of these procedures during health care provision. ${ }^{7}$

Most users stated that the received care was good or very good in spite of the barriers to continued care. On the other hand, $30.5 \%$ of those receiving care in accident and emergency units considered it to be regular/poor/very bad and this was the worst service in the opinion of respondents. This degree of dissatisfaction was higher than that found by PNADs in 2008 (13.5\%), 2003 (14.0\%) and $1998(2.4 \%)^{\mathrm{e}, \mathrm{h}}$ for any kind of care. For satisfied users, satisfaction is associated with service use, i.e., those who

h Instituto Brasileiro de Geografia e Estatística. PNAD - Pesquisa Nacional por Amostra de Domicílios - PNAD: acesso e utilização dos serviços de saúde 1998. Rio de Janeiro (RJ); 2000 [cited 2014 Dec 8]. Available from: http://www.ibge.gov.br/home/estatistica/populacao/ trabalhoerendimento/pnad98/saude/saude.pdf 
have access to services and for whom this experience is positive, tend to use services more. ${ }^{13}$ Most individuals who manage to use health services feel satisfied, despite significant inequities.

User satisfaction can be understood based on conceptual models. It may be limited to a checklist or be observed from the perspective of the theory of discrepancy, whereby levels of user satisfaction are predicted from the difference between expectations and the perception of the experience the user has. Furthermore, the theory of embodiment states that satisfaction is the difference between what is desired and what is obtained. ${ }^{8}$

People seek emergency service care owing to greater assurance of access (24h care - "open doors"), problem-solving and being able to have examinations and tests then and there. ${ }^{2,6}$

The degree of user satisfaction, apart from being an important indicator of health service quality and access, is also a tool that enables service managers to evaluate and monitor the health system. ${ }^{12}$ Problems affected by barriers to access can be less severe. However, when comparing this with the answers given by those to whom care was provided, no significant differences were found.

In a context of high prevalence of chronic diseases among adults, most of them seek care in order to cope with their health problems rather than to cure them. Therefore, regular contact with a health service provider can influence behavioural change and better treatment adherence, aim of staying healthy and having better quality of life. ${ }^{4, i}$

\section{REFERENCES}

1. Andersen RM, Yu H, Wyn R, Davidson PL, Brown ER, Teleki S. Access to medical care for low-income persons: how do communities make a difference? Med Care Res Rev. 2002;59(4):384-411. DOI:10.1177/107755802237808

2. Aranaz Andrés JM, Martínez Nogueras R, Gea Velázques de Castro MT, Teresa M, Rodrigo Bartual V, Antón García P, Gómez Pajares F. ¿Por qué los pacientes utilizan los servicios de urgencias hospitalarios por iniciativa propia? Gac Sanit. 2006;20(4):311-5.

3. Bastos GAN, Santos IS, Costa JSD, Capilheira MF. Uso de serviços ambulatoriais nos últimos 15 anos: comparação de dois estudos de base populacional. Rev Bras Epidemiol. 2011;14(4):620-32. DOI:10.1590/S1415-790X2011000400009
There are few studies describing and quantifying barriers to accessing health care and its continuity. This study analyzed when respondents last received health care, which varied between three months and one year. These periods were used to minimize bias in the respondents' recollections of what happened. ${ }^{3}$ Other positive features are the low percentage of subjects lost to the study in relation to the estimated sample size, sample size and comprehensiveness, and its being nationally representative, as well as its use of primary data.

The low proportion of individuals without access to health services and care hampered the precision of the analysis. Stratified or adjusted analysis could help to understand the most affected social groups. It can also contribute to the adoption of measures and policies to reduce inequalities, in addition to increasing the efficiency of the health system and scaling up access to services and their quality. Despite the low prevalence rates found, when these proportions are projected and applied to the entire Brazilian adult population living in urban areas, many individuals do not access the health system or have continued health care.

The importance of the access and continuity of health services is increasing in national and international literature. Most studies defines service use as a synonym of access and do not investigate adequately lack of access and continuity of care.

Quantifying lack of access and continuity of health actions and service supply throughout the national territory helps its magnitude to be recognized, assisting in the definition of strategies for controlling them in the health system.
4. Campos MO, Rodrigues Neto JF. Doenças crônicas não transmissíveis: fatores de risco e repercussão na qualidade de vida. Rev Baiana Saude Publica. 2009;33(4):561-81.

5. Carret MLV, Fassa ACG, Domingues MR. Inappropriate use of emergency services: a systematic review of prevalence and associated factors. Cad Saude Publica. 2009;25(1):7-28. DOI:10.1590/S0102-311X2009000100002

6. Carret MLV, Fassa AG, Paniz VMV, Soares PC. Características da demanda do serviço de saúde de emergência no Sul do Brasil. Cienc Saude Coletiva. 2011;16(Supl 1):1069-79. DOI:10.1590/S1413-81232011000700039

7. Cunha EM, Giovanella L. Longitudinalidade/ continuidade do cuidado: identificando dimensões e variáveis para a avaliação da Atenção Primária no contexto do sistema público de saúde brasileiro. Cienc Saude Coletiva. 2011;16(Supl 1):1029-42. DOI:10.1590/S1413-81232011000700036

\footnotetext{
' Macinko J, Dourado I, Guanais FC. Doenças crônicas, atenção primária e desempenho dos sistemas de saúde: diagnósticos, instrumentos e intervenções. Washington (DC): Banco Interamericano de Desenvolvimento; 2011 [cited 2015 Feb 13]. (Textos para Debate). Available from: http://apsredes.org/site2012/wp-content/uploads/2012/06/Publica\%C3\%A7\%C3\%A3o-BID.pdf
} 
8. Esperidião MA; Trad LA Bonfim. Avaliação de satisfação de usuários: considerações teórico-conceituais. Cad Saude Publica. 2006;22(6):1267-76. DOI:10.1590/S0102-311X2006000600016

9. Facchini LA, Piccini RX, Tomasi E, Thumé E, Teixeira VA, Silveira DS, et al. Avaliação de efetividade da Atenção Básica à Saúde em municípios das regiões Sul e Nordeste do Brasil: contribuições metodológicas. Cad Saude Publica. 2008;24(Supl 1)):s159-72. DOI:10.1590/S0102-311X2008001300020

10. Haggerty JL, Reid RJ, Freeman GK, Starfield BH, Adair CE, McKendry R. Continuity of care: a multidisciplinary review. BMJ. 2003;327(7425):1219-21. DOI:10.1136/bmj.327.7425.1219

11. Hill AP, Freeman GK. Promoting continuity of care in general practice (UK). London: Royal College of General Practitioners; 2011.

12. Massote AW, Lima AMD, Van Stralen CJ, Álvares J, FleuryTeixeira P, Belisário SA, et al. A contribuição de estudos de percepção da população usuária na avaliação da atenção básica. In: Hartz ZMA, Felisberto E, Silva LMV, organizadores. Meta-avaliação da atenção básica à saúde: teoria e prática. Rio de Janeiro: Editora Fiocruz; 2008. p. 410.

13. Mendoza-Sassi R, Béria JU. Utilización de los servicios de salud: una revisión sistemática sobre los factores relacionados. Cad Saude Publica. 2001;17(4):819-32. DOI:10.1590/S0102-311X2001000400016

14. Novaes HMD. Epidemiologia e avaliação em serviços de atenção médica: novas tendências na pesquisa. Cad Saude Publica. 1996;12(Supl 2):S7-12. DOI:10.1590/S0102-311X1996000600002

15. Paim J, Travassos C, Almeida C, Bahia L, Macinko J. The Brazilian health system: history, advances, and challenges. Lancet. 2011;377(9779):1778-97. DOI:10.1016/S0140-6736(11)60054-8

16. Pereira MG. Serviços de saúde. In: Pereira MG. Epidemiologia: teoria e prática. 6.ed. Rio de Janeiro: Guanabara Koogan; 2001. p.513-37.
17. Szwarcwald CL, Damacena GN. Amostras complexas em inquéritos populacionais: planejamento e implicações na análise estatística dos dados. Rev Bras Epidemiol. 2008;11(Supl 1):38-45. DOI:10.1590/S1415-790X2008000500004

18. Travassos C, Castro MSM. Determinantes e desigualdades sociais no acesso e na utilização de serviços de saúde. In: Giovanella L, Escorel S, Lobato LVC, Noronha JC, Carvalho Al, organizadores. Políticas e sistema de saúde no Brasil. Rio de Janeiro: Editora Fiocruz; 2008. p.215-46.

19. Travassos C, Martins M. Uma revisão sobre os conceitos de acesso e utilização de serviços de saúde. Cad Saude Publica. 2004;20(Supl 2):S190-8. DOI:10.1590/S0102-311X2004000800014

20. Travassos C, Oliveira EXG, Viacava F. Desigualdades geográficas e sociais no acesso aos serviços de saúde no Brasil: 1998 e 2003. Cienc Saude Coletiva. 2006;11(4):975-86. DOI:10.1590/S1413-81232006000400019

21. Turci MA, Lima-Costa MF, Proietti FA, Cesar CC, Macinko J. Intraurban differences in the use of ambulatory health services in a large Brazilian city. J Urban Health. 2010;87(6):994-1006. DOI:10.1007/s11524-010-9499-4

22. Vázquez ML, Silva MRF, Campos ES, Arruda IKG, Diniz AS, Veras IL, et al. Participação social nos serviços de saúde: concepções dos usuários e líderes comunitários em dois municípios do Nordeste do Brasil. Cad Saude Publica. 2003;19(2):579-91. DOI:10.1590/S0102-311X2003000200025

23. Victora CG, Barreto ML, Leal MC, Monteiro CA, Schmidt MI, Paim J, et al. Health conditions and health-policy innovations in Brazil: the way forward. Lancet. 2011;377(9782):2042-53. DOI:10.1016/S0140-6736(11)60055-X

Research supported by the Brazilian Ministry of Health (Process 23110.010118/2007-89).

Basead on the doctoral thesis of Alitéia Santiago Dilélio, titled: "Acesso e Utilização da Assistência Médica Ambulatorial ao Adulto na Rede de Serviços de Saúde do Brasil", presented to the Graduate Program in Epidemiology of Universidade Federal de Pelotas, in 2014.

The authors declare no conflict of interest. 